Gut and Liver, Vol. 10, No. 1, January 2016, pp. 10-11

\title{
Reappraisal of Antihypertensive Medicine Doxazosin and Carvedilol as a Potential Therapeutic for Hepatic Fibrosis
}

\author{
Yong-Han Paik ${ }^{*}+$ \\ ${ }^{*}$ Department of Medicine, Samsung Medical Center, Sungkyunkwan University School of Medicine, and ${ }^{\dagger}$ Department of Health Sciences and \\ Technology, Samsung Advanced Institute for Health Sciences and Technology, Sungkyunkwan University, Seoul, Korea
}

See "Doxazosin Treatment Attenuates Carbon Tetrachloride-Induced Liver Fibrosis in Hamsters through a Decrease in Transforming Growth Factor $\beta$ Secretion” by Martin Humberto Muñoz-Ortega, et al. on page 101, Vol. 10. No. 1, 2016

Liver cirrhosis results from chronic liver injuries that cause the accumulation of extracellular matrix and progression of hepatic fibrosis. ${ }^{1}$ The patients with liver cirrhosis may develop decompensation such as ascites, variceal bleeding, hepatic encephalopathy leading to death, in addition, patients having cirrhosis have a high risk for developing hepatocellular carcinoma. With the advent of potent oral antivirals against hepatitis B or $\mathrm{C}$ virus, even decompensated liver cirrhosis can be improved to the compensated cirrhosis. However, until now there was no direct antifibrotic therapy approved to treat hepatic fibrosis.

Hepatic fibrogenesis is a dynamic process and hepatic stellate cell (HSC) is a main fibrogenic cell type in the development of hepatic fibrosis. ${ }^{1}$ Various liver injuries from alcohol, hepatitis virus, and toxic bile acids, and so forth, activate and transform quiescent HSCs into activated HSCs. Activated HSCs proliferate, migrate, secrete inflammatory cytokines and produce type I collagen. HSCs mediate fibrogenic signaling pathways interacting with other cell types including hepatocytes, Kupffer cells, sinusoid endothelial cells, and infiltrating immune cells. The dynamic interactions between HSCs and various immune cells and other resident liver cells may result in the progression or regression of hepatic fibrosis. Activation of HSCs is promoted by interactions with hepatocytes, macrophages, innate lymphoid cells, B cells, natural killer T cells, and sinusoid endothelial cells.

Due to increasing understanding the molecular mechanisms of hepatic fibrogenesis, multiple candidates of antifibrotic therapeutics have been emerging. ${ }^{2}$ One useful strategy may be adopting agents that are approved for the treatment of other disease. This strategy can give us the advantage that safety of the drug and drug to drug interactions has been well characterized. In this regard, candesartan, an antihypertensive medicine blocking angiotensin II type I receptor, induced an improvement of hepatic fibrosis in patients with alcoholic liver disease. ${ }^{3}$ MuñozOrtega et al. ${ }^{4}$ in this issue of Gut and Liver explored the ant fibrotic effect of adrenergic receptor antagonist, doxazosin and carvedilol, that were commonly used as antihypertensive medicine, in rodent models of hepatic fibrosis. They found a decrease in type I collagen deposition and transforming growth factor $\beta$ (TGF- $\beta$ )-secreting cells in the hamster liver after co-administration with doxazosin and carvedilol in $\mathrm{CCl}_{4}$-induced hepatic fibrosis.

Doxazocin is a $\alpha 1$-adrenergic receptor antagonist and carvedilol is nonselective $\beta$-blocker with $\alpha 1$-adrenergic blocking activity that is used as antihypertensive drugs. Carvedilol is effective in reducing portal pressure and is used for primary and secondary prophylaxis in patients with high-risk esophageal varices. It was reported that HSCs express functional adrenoreceptor, and sympathetic nervous system neurotransmitters induce activation of HSCs., ${ }^{5,6}$ Activated HSCs in culture express $\alpha 1 \mathrm{~A}, \beta 1, \beta 2$ and, $\beta 3$ adrenoreceptors. HSCs synthesize and release the catecholamine to regulate HSCs basal growth, and prazosin or propranolol reduces HSCs basal growth. ${ }^{7}$ In addition, norepinephrine stimulates calcium spike and secretion of inflammatory chemokines such as regulated on activation, normal T cell expressed and secreted and interleukin-8 in HSCs. ${ }^{6}$ Norepinephrine and epinephrine stimulates HSCs through p38 mitogen-activated protein kinases, phosphoinositide 3-kinase, and mitogen-activated protein kinase kinase. Moreover, nor-

Correspondence to: Yong-Han Paik

Division of Gastroenterology and Hepatology, Department of Medicine, Samsung Medical Center, Sungkyunkwan University School of Medicine, 81 Irwon-ro, Gangnam-gu, Seoul 06351, Korea

Tel: +82-2-3410-3878, Fax: +82-2-3410-6983, E-mail: yh.paik@skku.edu

pISSN 1976-2283 eISSN 2005-1212 http://dx.doi.org/10.5009/gnl15576

(a) This is an Open Access article distributed under the terms of the Creative Commons Attribution Non-Commercial License (http://creativecommons.org/licenses/by-nc/4.0) which permits unrestricted non-commercial use, distribution, and reproduction in any medium, provided the original work is properly cited. 
epinephrine induces TGF- $\beta$ expression and type I collagen gene expression in HSCs. ${ }^{7}$ Taken together, adrenergic antagonists may be a candidate as a novel therapeutic for hepatic fibrosis.

However, caution is required when using antiadrenergic agents in patients with severe hepatic impairment. Many drugs are metabolized in the liver by enzymatic conversion into active, inactive or even toxic metabolites. The influence of liver disease status have on hepatic metabolism can be highly variable and unpredictable. ${ }^{8}$ Carvedilol and prazosin are metabolized by the liver, and it is recommended that these drugs not be administered to patients with clinically manifested hepatic impairment. The newer-generation of $\beta$-blockers such as carvedilol has a potent portal hypotensive effect that is superior to propranolol; however, it has greater risk to cause systemic hypotension. ${ }^{9}$ There has been debate regarding the safety and efficacy of nonselective $\beta$-blocker in patients with advanced cirrhosis with ascites. It was reported that use of nonselective $\beta$-blocker increase risks for hepatorenal syndrome and acute kidney injury reducing transplant-free survival in cirrhotic patients with spontaneous bacterial peritonitis. ${ }^{10}$ However, recent study reported that nonselective $\beta$-blocker are safe and associated with less waitlist death in patients with ascites complicating end-stage liver disease. ${ }^{11}$ Because antifibrotic therapy usually takes long duration of treatment, safety as well as efficacy is very important. Although in vitro and in vivo animal studies demonstrated the antifibrotic effect of adrenergic antagonist, the optimal dosing in cirrhotic patients should be further studied for the clinical trials in human.

\section{CONFLICTS OF INTEREST}

No potential conflict of interest relevant to this article was reported.

\section{REFERENCES}

1. Paik YH, Kim J, Aoyama T, De Minicis S, Bataller R, Brenner DA.
Role of NADPH oxidases in liver fibrosis. Antioxid Redox Signal 2014;20:2854-2872.

2. Mehal WZ, Schuppan D. Antifibrotic therapies in the liver. Semin Liver Dis 2015;35:184-198.

3. Kim MY, Cho MY, Baik SK, et al. Beneficial effects of candesartan, an angiotensin-blocking agent, on compensated alcoholic liver fibrosis: a randomized open-label controlled study. Liver Int 2012;32:977-987.

4. Muñoz-Ortega MH, Llamas-Ramírez RW, Romero-Delgadillo NI, et al. Doxazosin treatment attenuates carbon tetrachloride-induced liver fibrosis in hamsters through a decrease in transforming growth factor beta secretion. Gut Liver 2016;10:101-108.

5. Oben JA, Roskams T, Yang S, et al. Hepatic fibrogenesis requires sympathetic neurotransmitters. Gut 2004;53:438-445.

6. Sancho-Bru P, Bataller R, Colmenero J, et al. Norepinephrine induces calcium spikes and proinflammatory actions in human hepatic stellate cells. Am J Physiol Gastrointest Liver Physiol 2006;291:G877-G884.

7. Sigala B, McKee C, Soeda J, et al. Sympathetic nervous system catecholamines and neuropeptide $\mathrm{Y}$ neurotransmitters are upregulated in human NAFLD and modulate the fibrogenic function of hepatic stellate cells. PLoS One 2013;8:e72928.

8. Sokol SI, Cheng A, Frishman WH, Kaza CS. Cardiovascular drug therapy in patients with hepatic diseases and patients with congestive heart failure. J Clin Pharmacol 2000;40:11-30.

9. Bañares R, Moitinho E, Matilla A, et al. Randomized comparison of long-term carvedilol and propranolol administration in the treatment of portal hypertension in cirrhosis. Hepatology 2002;36: 1367-1373.

10. Mandorfer M, Bota S, Schwabl P, et al. Nonselective beta blockers increase risk for hepatorenal syndrome and death in patients with cirrhosis and spontaneous bacterial peritonitis. Gastroenterology 2014;146:1680-1690.e1.

11. Leithead JA, Rajoriya N, Tehami N, et al. Non-selective beta-blockers are associated with improved survival in patients with ascites listed for liver transplantation. Gut 2015;64:1111-1119. 